\title{
OCT Y FOTOTOXICIDAD MACULAR
}

\section{OPTICAL COHERENCE TOMOGRAPHY AND MACULAR PHOTOTOXICITY}

\author{
RODRÍGUEZ-MARCO NA ${ }^{1}$, ANDONEGUI-NAVARRO J ${ }^{1}$, COMPAINS-SILVA E ${ }^{2}$, \\ REBOLLO-AGUAYO A ${ }^{1}$, ALISEDA-PÉREZ-DE-MADRID D ${ }^{3}$, ARANGUREN-LAFLIN ${ }^{1}$
}

\section{RESUMEN}

Caso clínico: Se realiza una exploración ocular y tomografía de coherencia óptica (OCT) en tres pacientes con lesiones fototóxicas retinianas. Las angiografías fluoresceínicas muestran un defecto ventana. La OCT muestra hiporreflectividad en la porción externa de la fóvea y fragmentación de las capas más internas entre la porción interna de los fotorreceptores y los segmentos externos.

Discusión: Las lesiones retinianas tras exposición a la luz aparecen precozmente mostrando diferentes patrones en la OCT. La OCT sugiere que la disminución de visión asocia una lesión de fotorreceptores y epitelio pigmentario retiniano (EPR). La OCT es útil para objetivar la retinopatía fototóxica donde los cambios oftalmoscópicos pueden estar ausentes o ser mínimos.

Palabras clave: Fototoxicidad, retinopatía solar, eclipse solar, retinitis foveomacular, tomografía de coherencia óptica, luz del microscopio.

\begin{abstract}
Case report: Ocular examinations and optical coherence tomography (OCT) were performed in three patients with retinal phototoxicity lesions. Fluorescein angiography depicted a window defect. OCT exhibited hyporeflectivity at the outer foveal retina and fragmentation of the inner reflective layers, corresponding to the junction between the inner and outer photoreceptor segments.

Discussion: Retinal damage after light exposure has a rapid onset and shows different patterns in OCT examination. OCT findings suggest that decreased visual acuity may be associated with fullthickness photoreceptors and retinal pigment epithelium (RPE) involvement. OCT is a useful tool for objective assessment of retinal pathology in phototoxicity cases where fundus changes may be minimal or absent (Arch Soc Esp Oftalmol 2008; 83: 267-272).
\end{abstract}

Key words: Phototoxicity, solar retinopathy, solar eclipse, foveomacular retinitis, Optical coherence tomography, microscope light.

\footnotetext{
Recibido: 8/1/07. Aceptado: 3/3/08.

Servicio de Oftalmología. Hospital de Navarra. Pamplona. España.

1 Licenciado en Medicina.

2 Licenciado en Medicina. Hospital Virgen del Camino.

3 Doctor en Medicina. 


\section{INTRODUCCIÓN}

La maculopatía fototóxica (MF) es una alteración retiniana que aparece en pacientes cuya fóvea ha estado expuesta durante un tiempo prolongado a una fuente lumínica intensa. Generalmente afecta a pacientes tras observar un eclipse solar sin protección. Muchos de estos afectados suelen presentar problemas psicológicos, consumo de drogas (LSD) o practicas de misticismo. También, se han descrito lesiones similares después de mirar arcos de soldadura sin caretas de protección, punteros láser o incluso tras cirugía ocular produciéndose una reacción fotoquímica en los fotorreceptores. Los pacientes pueden notar un escotoma pericentral, metamorfopsia o pérdida de visión de leve a moderada entre una y cuatro horas tras la exposición. Oftalmoscópicamente aparece una pequeña lesión foveolar amarilla en forma de exudado o edema, seguida al cabo de unas dos semanas de pérdida de reflejo foveal, formación de acúmulo de pigmento y adelgazamiento de la fóvea. El escotoma puede disminuir a lo largo de unos meses y el paciente suele recuperar parte de la visión perdida $(1,2)$. Actualmente con la tomografía de coherencia óptica (OCT) podemos describir los daños a nivel intraretiniano ya que en un número elevado de casos las lesiones retinianas no se observan oftalmoscópicamente.

\section{CASO CLÍNICO}

\section{Caso 1}

Mujer de 60 años que acudió a la consulta por visión borrosa y sensación de mancha central en ambos ojos. Como antecedentes refería haber observado un eclipse solar dos meses antes. A la exploración presentaba agudeza visual (AV) de 0,5 en ambos ojos, presión intraocular y segmento anterior normal. En ambas máculas se apreciaban lesiones hipo e hiperpigmentadas redondeadas (fig. $1 \mathrm{~A}$ y B). La OCT demostró una pérdida en la capa del epitelio pigmentario retiniano (EPR). El resto de las capas retinianas no presentaban alteraciones (fig. 1 C y D). La angiografía fluoresceínica (AGF) mostraba de forma precoz zonas hiperfluorescentes en anillo (fig. $1 \mathrm{E}$ ).

\section{Caso 2}

Varón de 39 años intervenido de pterigion en el ojo derecho (OD) en dos ocasiones en los últimos 6 meses, con un tiempo aproximado de las dos cirugías de una hora y media. Antes de la intervención la $\mathrm{AV}$ era de la unidad en ambos ojos y el segmento anterior y posterior fueron normales. En la última revisión el paciente se quejaba de disminución de $\mathrm{AV}$ en OD con dificultad para la lectura y metamorfopsia. La AV era de 0,4 con OD y la unidad con ojo izquierdo (OI). En fondo de ojo se apreciaba una lesión redondeada hipopigmentada en área macular (fig. 2 A). La AGF mostraba una zona hiperfluorescente de aparición precoz (fig. 2 B). En la OCT aparecía un desprendimiento del EPR (fig. 2 C).

\section{Caso 3}

Varón de 55 años diagnosticado de cataratas nucleares ++ en ambos ojos e intervenido de facoemulsificación más lente intraocular hacía dos meses en OD. Tras haber estado tomando el sol (reconocía haberse quedado dormido una hora) notó disminución de AV en el OD. En la mácula se apreciaba una lesión amarillenta (fig. 2 D). En la OCT aparecía un engrosamiento de las capas retinianas, rotura del EPR y leve desprendimiento seroso de retina, con una señal hiperreflectiva a nivel macular (fig. 2 E). En el OI no se apreciaban lesiones retinianas.

\section{DISCUSIÓN}

La MF es resultado de una lesión fotoquímica y térmica de la retina por la radiación ultravioleta, bien procedente de la luz solar o del microscopio, y se caracteriza por afectar a las capas más externas de la retina. Está relacionada con la intensidad, tiempo de exposición y longitud de onda de la fuente lumínica, siendo la luz azul y ultravioleta (longitud de onda menor a 300-350 nm) la más dañina para el ojo (3-5). Clínicamente se caracteriza por una lesión amarillenta a nivel foveal, un defecto ventana en la AGF y un escotoma central o paracentral que con el tiempo disminuye. Las lesiones amarillentas iniciales son sustituidas posteriormente por un moteado del EPR o incluso un agujero lamelar. Histológicamente la fase aguda de la lesión muestra daño del EPR con desprendimiento, necro- 


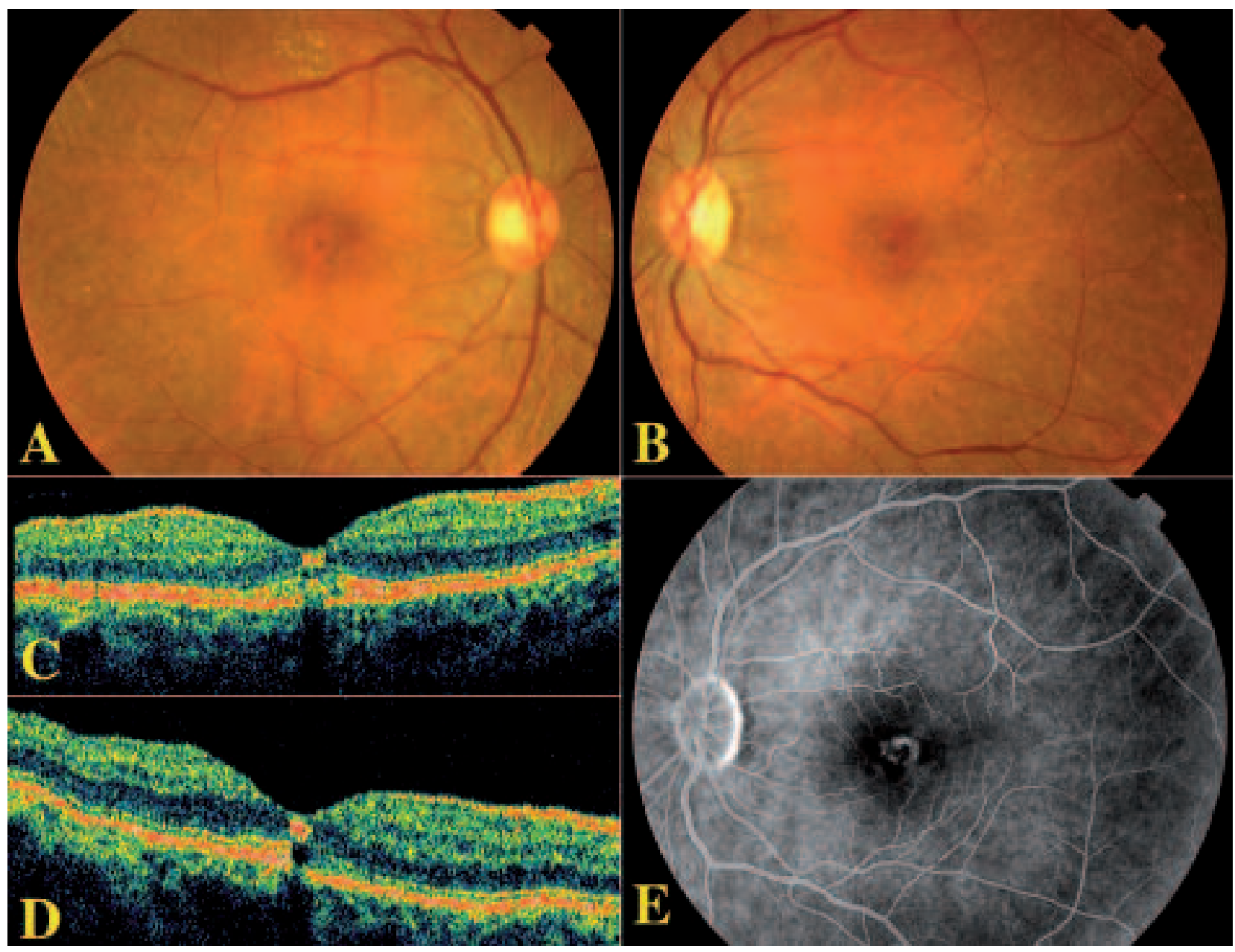

Fig. 1: A y B: Retinografía de OD y OI mostrando una retinopatía solar cicatrizada caracterizada por áreas hiperpigmentadas rodeada de un halo hipopigmentado. C y D: OCT de OD y OI mostrando una pérdida local de células en la capa del EPR. E: AGF de OI en la que se aprecia un defecto ventana a nivel foveal, con los mismos hallazgos en $O D$.

sis, pigmentación irregular y cambios en los fotorreceptores $(1,2)$. Aquellos pacientes con medios transparentes, afáquicos, emétropes y bajos hipermétropes parecen tener un riesgo mayor de desarrollar MF al tener mayor capacidad de concentrar la luz sobre la retina y mácula.

Es importante destacar que en la mitad de los casos la lesión foveal no se puede apreciar oftalmoscópicamente pese a existir una clínica manifiesta. En estos casos la OCT es de gran ayuda para mostrar cambios sutiles donde las técnicas convencionales (oftalmoscopía y AGF) no nos ayudan a delimitar las lesiones in vivo. La OCT muestra una zona hiperreflectiva que generalmente afecta a todas las capas de la fóvea sin apreciarse edema subyacente y conservando la mácula un contorno normal, pudiendo relacionarse el tamaño y localización de la lesión en la OCT con la visión fundoscópica. Otras veces se observa integridad de las capas más internas y disrupción del EPR bajo la fovea sugiriendo un agujero lamelar $(1,2)$. Sin embargo las alteraciones estructurales vistas en la mácula por OCT no son iguales en todos los ojos, ni en todos los trabajos publicados $(1,2,4)$ por lo que han de existir factores externos, como la intensidad y duración de la exposición y factores individuales que hacen tan heterogéneas las lesiones. Tal vez el único hallazgo constante en los diferentes trabajos sea la pérdida focal de EPR y la hiporeflectividad de las capas internas de la retina. 


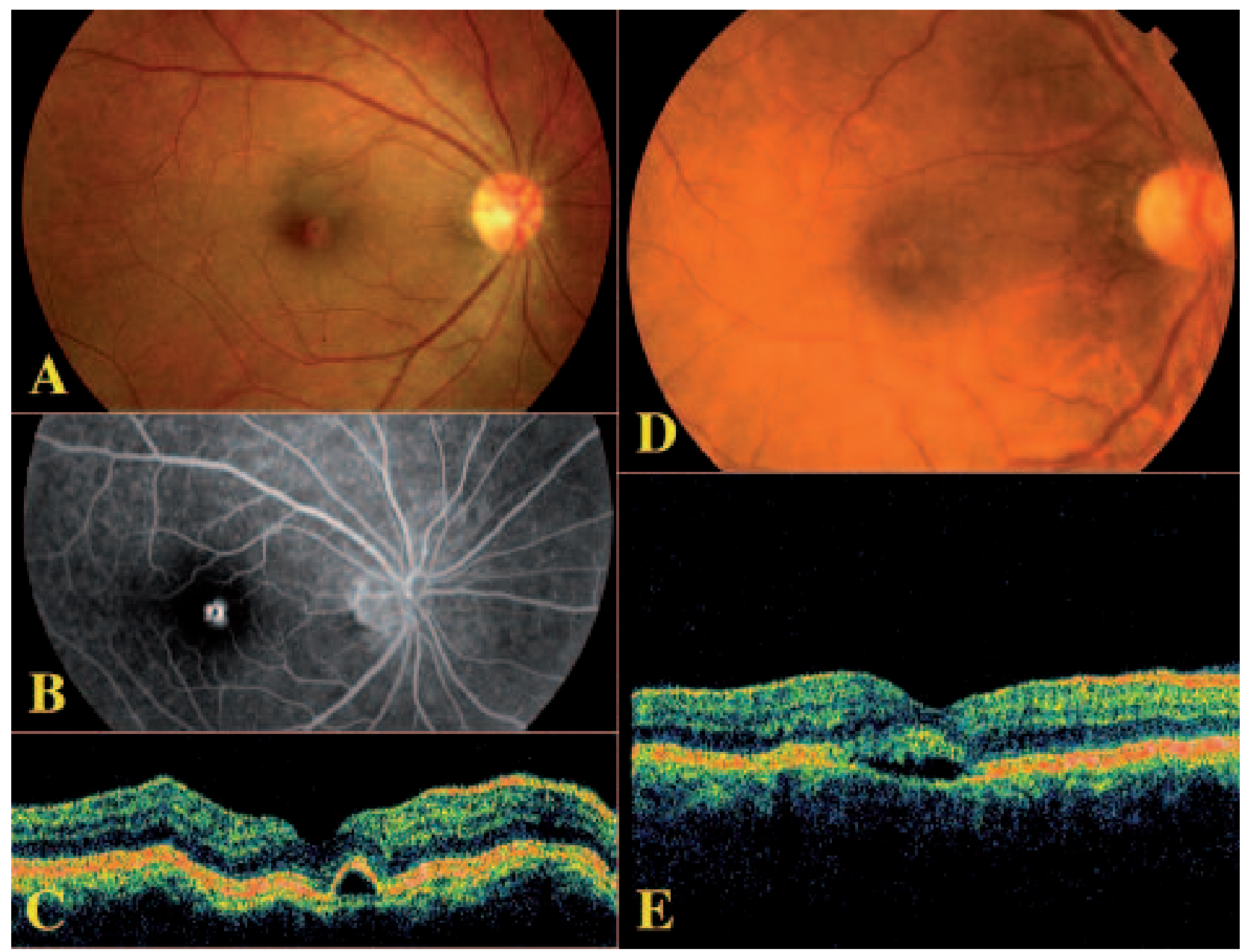

Fig. 2: A: Lesión redondeada e hipopigmentada en área macular. B: Áreas hiperfluorescentes de aparición precoz. C: Desprendimiento de EPR que se mantiene en tiempo. D: Lesión redondeada e hipopigmentada en área macular. E: Rotura del EPR e hiporefletividad de las capas externas que representa un leve desprendimiento seroso de retina.

Bechmann et al. (2) señalan que las alteraciones hiperreflectivas retinianas aparecen precozmente tras la exposición a la luz solar, desapareciendo las mismas en el primer mes excepto las lesiones del EPR. En nuestros dos casos de maculopatía solar este defecto se mantiene incluso un año después y en el caso de desprendimiento del EPR del paciente número dos permanece sin cambios. En todos ellos la AV es la misma desde la primera visita. Imágenes similares en el examen de fondo de ojo nos las podrían dar patologías como la corioretinopatía serosa central, pero en estos casos no coincidirían con las imágenes clásicas en humo de chimenea o en mancha de tinta de la angiografía fluoresceínica. Tampoco existen signos de neovasculariza- ción subretiniana o rotura del EPR en el que veríamos dos zonas claramente diferenciadas con una línea bien definida correspondiente al borde del EPR replegado. También excluimos focos infecciosos al no observarse vitritis, retinitis o coroiditis y mantenerse la lesión estable con el paso del tiempo. En el segundo caso establecemos diagnóstico diferencial con la distrofia foveomacular viteliforme del adulto, pero en nuestro paciente la presentación no es bilateral y en la OCT no se identifica depósito de material entre el EPR y la membrana de Bruch en forma de una banda hiperreflectiva.

El pronóstico visual de la retinopatía solar es generalmente favorable y la AV mejora espontáneamente por lo que el uso de corticoides ha sido 
cuestionado, pero la educación de la sociedad, la prevención y la profilaxis en las intervenciones quirúrgicas usando la mínima intensidad de luz posible, interponer elementos opacos en el área pupilar, usar iluminación indirecta oblicua o intercalar filtros en cirugías de segmento anterior son fundamentales para prevenir los daños.

En conclusión, la OCT puede ser una herramienta útil para verificar, caracterizar y realizar un seguimiento de la afectación retiniana en casos como los descritos donde a veces los cambios de fondo de ojo pueden estar ausentes o ser mínimos. Los hallazgos en la OCT sugieren que la disminución de visión se asocia con la lesión de los fotoreceptores y EPR.

\section{BIBLIOGRAFÍA}

1. Garg SJ, Martidis A, Nelson ML, Sivalingam A. Optical coherence tomography of chronic solar retinopathy. Am J Ophthalmol 2004; 137: 351-354.

2. Bechmann M, Ehrt O, Thiel MJ, Kristin N, Ulbig MW, Kampik A. Optical coherence tomography findings in early solar retinopathy. Br J Ophthalmol 2000; 84: 547-548.

3. Andonegui Navarro J, Marcuerquiaga Arriaga J. Lesiones fototóxicas inducidas por la luz de xenon. Arch Soc Esp Oftalmol 2000; 75: 117-120.

4. Codenotti $M$, Patelli $F$, Brancato $R$. OCT findings in patients with retinopathy after watching a solar eclipse. Ophthalmologica 2002; 216: 463-466.

5. Khwarg SG, Linstone FA, Daniels SA, Isenberg SJ, Hanscom TA, Geoghegan $M$, et al. Incidence, risk factors, and morphology in operating microscope light retinopathy. Am J Ophthalmol 1987; 103: 255-263. 